\title{
On the Existence of Non-Intersecting Straight Lines on the Plane
}

\author{
Andrew Chubykalo1, Sergey Artekha² \\ ${ }^{1}$ Unidad Academica de Fisica, Universidad Autónoma de Zacatecas, Zacatecas, Mexico \\ ${ }^{2}$ Space Research Institute of RAS, Moscow, Russia \\ Email: achubykalo@yahoo.com.mx
}

How to cite this paper: Chubykalo, A. and Artekha, S. (2018) On the Existence of Non-Intersecting Straight Lines on the Plane. Journal of Modern Physics, 9, 816-819.

https://doi.org/10.4236/jmp.2018.95052

Received: March 7, 2018

Accepted: April 7, 2018

Published: April 10, 2018

Copyright (c) 2018 by authors and Scientific Research Publishing Inc. This work is licensed under the Creative Commons Attribution International License (CC BY 4.0).

http://creativecommons.org/licenses/by/4.0/

\begin{abstract}
In this brief note, we adduce the logical rationale that if at least one infinite straight line non-intersecting with the given straight line passes through a given point not lying on a given straight line, then it must be unique.
\end{abstract}

\section{Keywords}

Infinite Straight Line, Euclid's Fifth Postulate, Non-Euclidean Geometries

\section{Brief Historical Background}

One axiom of Euclidean geometry had bothered mathematicians somewhat, not because there was in their minds any doubt of its truth but because of its formulation. We have in mind the parallel postulate or, as it is often referred to, Euclid's fifth postulate. As Euclid worded it, it states:

If a straight line falling on two straight lines makes the interior angles on the same side less than two right angles, then the two straight lines if extended will meet on that side of the straight line on which the angles are less than two right angles [1].

Mathematicians regard that the parallel postulate in the form stated by Euclid was thought to be somewhat too complicated. Euclid apparently feared to assume that there could be infinite straight lines. Nevertheless Euclid did imply the existence of infinite straight lines for, were they finite, they could not be extended as far as necessary in any given context. So hereafter we will use the existence of infinite straight lines at an infinite plane and allude to the received version of the parallel postulate proposed by John Playfair in 1795 which states:

Through a given point $n$ not on a straight line $G$ (Figure 1) there is one and only one straight line $N$ in the plane of $n$ and $G$ which does not intersect with $G$ [2]. 


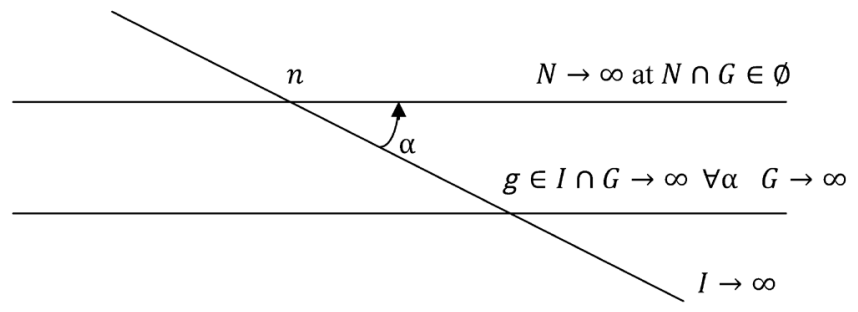

Figure 1. The geometrical proof of the theorem for non-intersecting straight lines.

In our work, we will try to avoid using the word-group "parallel straight lines" but will use the phrases "non-intersecting" and "intersecting" straight lines.

\section{The Existence of a Straight Line That Does Not Intersect with Another Straight Line}

Since we will use some common statement for the Euclidean and Lobachevsky geometries, let us now somewhat change the literal sense of the axiom of Playfair and try to prove the following statement:

We assume that there exists at least one infinite straight line $N$ in the plane of $n$ and $G$, which passes through a given point $n$ not lying on an infinite straight line $G$ and does not intersect with $G$ (i.e. $N \cap G \in \varnothing$ or $\nexists N \cap G$ ) (Figure 1). In this case this infinite straight line is the only one passing through the point $n$ that does not intersect with $G$.

We shall rely on the first postulate of Euclid (axiom of belonging). The following cases of mutual arrangement are possible for two arbitrary straight lines: they may not have common points at all, have one point of intersection or completely coincide. If two straight lines have two different common points, then these lines coincide (all points are common for them).

Admitting a hypothesis of the existence of infinite straight lines at an infinite plane, we adduce here the following reasoning. Let us move the infinite straight line $I$ which intersects two a priory mutually non-intersecting infinite straight lines $N$ and $G$ at the points $n$ and $g$, respectively (Figure 1 ), so that the point $n$ will be remained stationary, while the point $g$ would move to the right (for definiteness only; the opposite motion can be analyzed similarly). In order to straight lines $I$ and $G$ no longer intersect, their right ends must first be combined $^{1}$ then must be split. But, as it follows from the meaning of the word "infinite", these lines do not have ends, so, these non-existent ends cannot be combined and, accordingly, these straight lines cannot be split, that is cannot become disjoint (or non-intersecting)! Moreover, what happens to the angle $\alpha$ when the point $g$ moves to the right? The angle $\alpha$ will tend to zero (instead of moving, we also can choose an infinite sequence of points $g_{k}$ having a limit point $+\infty$; as a result, we obtain the limit point $\alpha_{k} \rightarrow 0$ ). But the angle $\alpha$ cannot reach zero

${ }^{1}$ We require here that the left (with respect to the point $n$ ) parts of these straight lines initially do not intersect. 
during the movement of the point $g$ along the straight line $G$, because in this case, the straight line $I$ which always (see our reasoning above) has to be a straight line intersecting the straight line $G$ on the right hand of the point $n$ certainly must coincide with the straight line $N$ non-intersecting with $G$ by definition.

Therefore, we can argue that if there is an infinite straight line having a point $n$ that is outside of another given straight line and does not intersect with this given straight line, then such a straight line is unique!

In other words, we can claim that we have proved the following theorem:

If at least one straight line $N$ in the plane $n$ and $G$ passes through the given point $n$ which is not on the straight line $G$ (Figure 1), and does not intersect with $G$, then such straight line $N$ is unique.

What is the difference between our theorem and the axiom of Playfair? After all, the rejection of this axiom (or the fifth postulate of Euclid, which is the same thing) led to the possibility of creating non-Euclidean geometries.

So, the of Playfair's axiom requires the existence of only one straight line passing through a point $n$ that does not intersect with a given another straight line, and our theorem states that if such a straight line exists, then it is necessarily the only one!

Note that our theorem is proved in the framework of Euclidean geometry without using the of Playfair's axiom. This means that the rejection of the fifth postulate of Euclid (or Playfair's axiom) cannot lead to the creation of a non-Euclidean geometry of Lobachevsky (hyperbolic geometry), because Lobachevsy's geometry admits an infinite number of straight lines passing through a point $n$ nonintersecting with the line $G$.

On the contrary, our theorem does not prevent the existence of non-Euclidean geometries of Riemann (now called double elliptic geometry) and Felix Klein (now called single elliptic geometry), ${ }^{2}$ taking into account this statement from the theorem: "If at least one straight line $N$ in the plane $n$ and $G$ passes through the given point $n$ which is not on the straight line $G$, and does not intersect with G...”.

Here we should recall that there is the following well-known theorem (see, e.g., [3] [4] and [5] or Lobachevsky himself [6]): “Lobachevsky's geometry is consistent if and only if Euclidean geometry is consistent". For its proof, models of one of geometries in another one are used. However, such a proof is not direct (since some mistaken suppositions can be mutually compensated that can lead to the seemingly realized models). One and the same mathematical object can be simultaneously placed and described in the space of Euclidean geometry and Lobachevsky geometry. Moreover, in Euclidean geometry there are no singular points and singular line (boundary), all points of space are included in it, but, in the Lobachevsky's geometry, on the contrary, lines and points beyond the special

${ }^{2}$ All straight lines intersect in two points in the Riemann's geometry and in one point in the Klein's geometry. 
boundary are not defined. Such local models can exist in the framework of Euclidean geometry with additional conditions and definitions only.

\section{Conclusions}

Thus, the following theorem was proved: in the framework of Euclidean geometry, if there exists some straight line passing through a point $n$ that does not intersect with a given another straight line, then it is necessarily the only one straight line.

So, on the basis of our theorem, proved in the framework of Euclidean geometry without referring to the fifth postulate of Euclid, we can definitely state that from so-called non-Euclidean geometries only geometries of the Riemann and Klein are permissible, but the non-Euclidean geometry of Lobachevsky remains outside of generally accepted mathematics.

\section{References}

[1] Euclid (2002) Euclid's Elements. Grin Lion Press, Lion.

[2] Dieudonné, J. (1970) The American Mathematical Monthly, 77, 134-135. https://doi.org/10.2307/2317325

[3] Bonola, R. (1955) Non-Euclidean Geometry. N. Y. Dover Publication, New York.

[4] Coxeter, H.S.M. (1965) Non-Euclidean Geometry. University of Toronto Press, Toronto.

[5] Milnor, J.W. (1982) Bulletin of the American Mathematical Society, 6, 9-24. https://doi.org/10.1090/S0273-0979-1982-14958-8

[6] Lobachevsky, N.I. (1840) Geometrische Untersuchungen zur Theorie der Parallellinien. F. Fincke, Berlin. 